\title{
Cross-cultural Adaptation and Validation of the Dutch Version of SWAL-QoL
}

\author{
H. C. A. Bogaardt $\cdot$ R. Speyer $\cdot$ L. W. J. Baijens $\cdot$ \\ W. J. Fokkens
}

Published online: 15 July 2008

(C) The Author(s) 2008

\begin{abstract}
The aim of this work was to translate the 44-item SWAL-QoL into Dutch (SWAL-QoL-NL) and compare the validity of this questionnaire against Euroqol in a Dutch population with dysphagia. SWAL-QoL was translated according to international guidelines. SWAL-QoL-NL and Euroqol were completed by 152 patients in seven diagnosis groups. Internal consistency and correlations were calculated. Scores for nine subscales (General burden, Food selection, Eating duration, Fear of eating, Sleep, Fatigue, Mental health, Social functioning and a symptom score) ranged between 0.80 and 0.92 (Cronbach's $\alpha$ ). Two subscales (Eating desire and Communication scored 0.67 and 0.60 , respectively, and were removed from the questionnaire. The 14-item battery on clinical symptoms showed an internal consistency of 0.80 , allowing the use of a sum score on group level in clinical research. Correlation of SWALQoL-NL subscales with the Euroqol was negligible to low (Pearson's correlations range $=0.09-0.36$ ). The 39-item SWAL-QoL-NL proved to be a reliable tool to examine the impact of dysphagia on quality of life in a Dutch population. Internal consistency allows the use of nine subscales of SWAL-QoL-NL for comparisons on a group level $(0.80<$ $\alpha<0.92$ ) only. Also a Symptom score can be derived from the raw data.
\end{abstract}

H. C. A. Bogaardt $(\bowtie) \cdot$ W. J. Fokkens

Department of Otorhinolaryngology/D2-313, Academic Medical

Center, Meibergdreef 9, 1100 DD Amsterdam, The Netherlands

e-mail: h.c.bogaardt@amc.uva.nl

R. Speyer · L. W. J. Baijens

Department of ORL and Head and Neck Surgery,

University Hospital Maastricht, Maastricht, The Netherlands
Keywords Deglutition - Deglutition disorders · Dysphagia - Quality of life · Outcome measurements

A large amount of clinical research has been aimed at evaluating the impact of different diseases and health problems on the quality of life of patients. In the early 1990s over 160 different measures were used to assess health-related quality of life (HR-QoL) [1].

Eating and drinking are basic necessities of man. Problems with swallowing (dysphagia) therefore have severe consequences for the quality of life of patients [2]. In 2000 the SWAL-QoL was developed by McHorney et al. $[3,4]$ as a patient-based, dysphagia-specific tool to evaluate the impact of swallowing problems on the quality of life in patients with dysphagia. The SWAL-QoL was initially derived from a 185 item pool and scaled down to a 93-item questionnaire. Later this 93-item questionnaire was further reduced into two different questionnaires: the 44-item SWAL-QoL, a dysphagiaspecific quality-of-life questionnaire and the 15-item SWALCare, a questionnaire that evaluates dysphagia therapy from a patient's perspective [3]. SWAL-QoL consists of 10 qualityof-life concepts (General burden, Food selection, Eating duration, Eating desire, Fear of eating, Sleep, Fatigue, Communication, Mental health and Social functioning with a total of 30 items and a 14-item battery to provide clinical researchers and practitioners information on symptoms.

Cronbach's $\alpha$ coefficients [5] were used by the developers of SWAL-QoL to determine the internal consistency reliability of each of the ten subscales. According to McHorney et al. [6], coefficients ranged from 0.79 to 0.91 , allowing the SWAL-QoL to be used only for group-level research according to Bland and Altman [5]. Thus, SWAL-QoL also allows researchers to derive sum scores for each of the ten concepts from the raw data provided by the questionnaire. 
Since its first publication in 2000, SWAL-QoL has shown to be the gold standard in dysphagia research regarding quality-of-life issues of patients with dysphagia and is frequently used [7-9]. Although SWAL-QoL has been translated into and used in other languages, to date no clinimetrical evaluations have been made of these translations. In this study we therefore translated the SWAL-QoL into Dutch (SWAL-QoL-NL) and performed a cross-cultural adaptation and validation of SWAL-QoL in order to provide a clinimetrically valid tool for assessing the patients' perspectives of dysphagia treatment in the Netherlands. In our research design we compared SWAL-QoL-NL to Euroqol as a general quality-of-life assessment tool [10]. Because both questionnaires are instruments that measure quality of life, we hypothesized that some correlation between both instruments would be found. However, because the SWALQoL was derived from a very dysphagia-specific-item pool $[3,4]$ compared to general quality-of-life questions, we hypothesized that this correlation would not be strong.

\section{Respondents and Methods}

Translation of SWAL-QoL

The original SWAL-QoL was translated into the Dutch language according to the process of translation and backtranslation as described in international guidelines [1]. This process yielded the initial 44-item Dutch version of SWALQoL, called SWAL-QoL-NL. Next to the 44-item test battery of SWAL-QoL-NL, general questions on feeding status, marital status, education levels, and time-needed-to-complete SWAL-QoL-NL were evaluated to gather information on social background and the clinical use of SWAL-QoL-NL.

\section{Euroqol}

Euroqol is a standardized instrument for use as a measure of health outcome. Applicable to a wide range of health conditions and treatments, Euroqol provides a simple descriptive profile and a single index value for health status. Euroqol is designed for self-completion by respondents and is developed for use in postal surveys, clinics, and face-to-face interviews [10]. Since its introduction in 1990, Euroqol is frequently used to determine health status of patients in a wide variety of groups and different research projects [11-13].

Euroqol consists of a visual analog scale (also called the "thermometer") from 0 to 100 , on which the respondent marks his general well-being. Further, Euroqol consists of five short questions regarding five dimensions or constructs (hence, in literature the name EQ-5D is also used for Euroqol). These five explored constructs are Mobility, Self-care, Usual activities, Pain and Discomfort, and Anxiety and Depression.
Respondents

To establish a broad range of respondents, patients diagnosed with (oropharyngeal) dysphagia based on a variety of diagnoses were recruited from the outpatient clinics of two large university hospitals (Amsterdam and Maastricht). Also, a range of patients with oropharyngeal dysphagia in nursing home facilities across the Netherlands were asked to participate. All possible respondents were asked to complete both Euroqol and SWAL-QoL-NL.

Statistical Analysis and Considerations

For statistical analysis SPSS 12.0 (SPSS Inc, Chicago, IL, USA) was used. Internal consistency of the SWAL-QoLNL was calculated using Cronbach's $\alpha$. Correlations between the subscales of the SWAL-QoL-NL and the EQ5D were determined by calculating the Pearson's correlation coefficient $(r)$. According to Bland and Altman [5], Cronbach's $\alpha$ values between 0.7 and 0.8 were regarded as satisfactory for comparing on group level, and values of 0.9 and higher were considered to be needed for individual applications. For the correlation coefficients $(r)$, a minimum value for a strong correlation was set at 0.7 and above [14-16]. Correlation coefficients between 0.3 and 0.7 were considered to be a substantial correlation only, and $r<0.3$ was considered to be a weak correlation.

For additional analysis, all data were formally tested for normality with the Kolmogorov-Smirnoff test for normality. Based on normality, unpaired tests were used to explore differences in (sum) scores between different patient groups.

\section{Results}

SWAL-QoL-NL and Euroqol were completed by 152 respondents, who ranged in age from 19.7 to 91.2 years old $($ mean $=64.8 \pm 13.2)$ and 65\% were male. Respondents could be split into seven different diagnostic groups (stroke $28 \%$, Parkinson $24 \%$, neuromuscular diseases $9 \%$, other neurologic diseases $16 \%$, head and neck oncology $11 \%$, idiopathic cricopharyngeal problems $5 \%$, and unknown etiology 7\%). A vast majority of patients (133 of 152) were on an oral diet, $12.5 \%(n=19)$ was dependent on tube feeding. Of all respondents, $69.7 \%$ had at least a secondary-level education (high school) or higher. Over $60 \%$ were married or living with a partner. Respondents' characteristics are presented in Table 1.

Over $60 \%$ of all respondents received help to complete the questionnaires. This help mainly consisted of another person reading the questions and writing down the answers (83\%). In only 7 cases (7\%) somebody else answered the 
Table 1 Patients' characteristics $(n=152)$

\begin{tabular}{ll}
\hline Characteristic & Number \\
\hline Gender & \\
Male & $99(65 \%)$ \\
Female & $52(34 \%)$ \\
Missing & $1(1 \%)$ \\
Age & $64.8 \pm 13.2(19.7-91.2)^{\mathrm{a}}$ \\
Diagnosis & \\
Stroke & $43(28.3 \%)$ \\
Parkinson's disease & $36(23.7 \%)$ \\
Neuromuscular diseases & $13(8.6 \%)$ \\
Other neurologic diseases & $25(16.4 \%)$ \\
Head and neck oncology & $17(11.2 \%)$ \\
Cricopharyngeal problems & $8(5.3 \%)$ \\
Unknown & $10(6.6 \%)$ \\
Education & \\
Primary & $41(27.0 \%)$ \\
Secondary & $74(48.6 \%)$ \\
University & $32(21.1 \%)$ \\
Missing & $5(3.3 \%)$ \\
Marital status & \\
Single & $24(15.8 \%)$ \\
Married/cohabiting & $95(62.5) \%$ \\
Separated & $9(5.9 \%)$ \\
Widowed & $22(14.5 \%)$ \\
Missing & $2(1.4 \%)$ \\
Tube feeding & $19(12.5 \%)$ \\
Euroqol VAS score & $59.9 \pm 19.5(0-99)^{\mathrm{a}}$ \\
\hline &
\end{tabular}

${ }^{\mathrm{a}}$ Mean $\pm \mathrm{SD}$ (minimum-maximum)

questions for the respondent. For respondents who received help it took 22 minutes (range $=1-60$ ) to complete SWAL-QoL-NL; respondents who completed the questionnaire by themselves took fractionally less time: $20 \mathrm{~min}$ on average (range $=1-60$ ). Table 2 shows the clinical use of SWAL-QoL-NL.

For each of the ten subscales, the sum scores were derived from the questionnaires. Mean scores on the subscales ranged from 42.9 to 82.9 , where a score of " 0 " represents the worst possible score and "100" the most optimal score. Cronbach's $\alpha$ coefficients on the SWAL-
Table 3 Reliability coefficients of Dutch version of SWAL-QoL

\begin{tabular}{llllll}
\hline Subscale & $n$ & No. items & Mean & SD & Cronbach's $\alpha$ \\
\hline General burden & 152 & 2 & 47.3 & 31.6 & 0.84 \\
Food selection & 144 & 2 & 62.3 & 29.2 & 0.87 \\
Eating duration & 147 & 2 & 42.9 & 32.6 & 0.82 \\
Eating desire & 149 & 3 & 69.6 & 28.2 & 0.67 \\
Fear of eating & 149 & 4 & 82.9 & 21.1 & 0.83 \\
Sleep & 150 & 2 & 67.3 & 32.0 & 0.80 \\
Fatigue & 149 & 3 & 60.8 & 27.4 & 0.83 \\
Communication & 152 & 2 & 56.8 & 28.3 & 0.60 \\
Mental health & 152 & 5 & 66.8 & 26.9 & 0.89 \\
Social functioning & 149 & 5 & 58.0 & 31.1 & 0.92 \\
Symptoms & 137 & 14 & 62.4 & 18.3 & 0.80 \\
\hline
\end{tabular}

QoL-NL ranged from 0.60 to 0.92 on the ten subscales. In addition, a sum score and Cronbach's $\alpha$ were calculated for the 14-item symptom battery and was estimated to be 62.4 $( \pm 18.3)$ and 0.80 , respectively. Table 3 shows the clinimetrical characteristics of the SWAL-QoL-NL.

Mean score on the EQ-5D visual analog scale (VAS) was $59.9( \pm 19.5)$. On the Euroqol subscale Mobility, $62 \%$ of our patients reported a problem. Percentages of patients reporting any problems on the subscales Self-care, Usual activities, Pain/Discomfort and Anxiety/Depression were 53, 61, 45, and 30, respectively. The subscales Mobility and Self-care revealed statistically different scores compared with the other subscales of Euroqol (Kruskal-Wallis; $p<0.001)$. Table 4 shows the scores on Euroqol for our patient group.

Clinical validity of SWAL-QoL-NL was assessed by correlating the subscale scores on SWAL-QoL-NL with the five subscales and the VAS scores on Euroqol. Pearson's correlation coefficients ranged from -0.24 to 0.36 , showing in general a weak correlation between the two assessment tools. Significant (2-tailed) correlations at a 0.01 level were found between the Euroqol VAS score and three SWAL-QoL-NL subscales (Eating duration, Fatigue and Social effects. At a 0.05 level three further significant correlations were found. The correlations between the subscores on the SWAL-QoL-NL and the Euroqol are shown in Table 5.

Table 2 Clinical use of SWAL-QoL-NL

\begin{tabular}{llll}
\hline Was patient helped with questionnaire? & When helped, how? & $n$ & Time (min) \\
\hline No & & $58(38.2 \%)$ & $19.6 \pm 8.9(1-60)$ \\
Yes & & $94(61.8 \%)$ & $21.5 \pm 8.8(1-60)$ \\
& Read questions/wrote down answers & $83(83.3 \%)$ \\
& Caregiver answered for the patient & $7(7.4 \%)$ \\
& Other & $4(4.3 \%)$ \\
\hline
\end{tabular}


Table 4 Percentage of respondents with any problem on Euroqol

\begin{tabular}{lrccccc}
\hline Diagnosis & $n$ & Mobility & Self-care & $\begin{array}{l}\text { Usual } \\
\text { activities }\end{array}$ & $\begin{array}{l}\text { Pain/ } \\
\text { discomfort }\end{array}$ & $\begin{array}{l}\text { Anxiety/ } \\
\text { depression }\end{array}$ \\
\hline All patients & 115 & 61.8 & 52.6 & 61.2 & 44.7 & 29.6 \\
Stroke & 42 & 88.1 & 83.3 & 85.7 & 50.0 & 47.6 \\
Parkinson's disease & 6 & 83.3 & 83.3 & 83.3 & 50.0 & 50.0 \\
Neuromuscular diseases & 12 & 100.0 & 76.9 & 84.6 & 53.8 & 30.8 \\
Other neurologic diseases & 25 & 92.0 & 76.0 & 68.0 & 56.0 & 32.0 \\
Head and neck oncology & 14 & 50.0 & 30.8 & 92.9 & 71.4 & 35.7 \\
Esophageal problems & 7 & 57.1 & 28.6 & 28.6 & 100.0 & 28.6 \\
Unknown & 9 & 66.7 & 55.6 & 88.9 & 66.7 & 33.3 \\
$p$ value & & $0.003^{\mathrm{a}}$ & $0.000^{\mathrm{a}}$ & $0.194^{\mathrm{a}, \mathrm{b}}$ & $0.056^{\mathrm{a}, \mathrm{b}}$ & $0.128^{\mathrm{a}, \mathrm{b}}$ \\
\hline
\end{tabular}

Table 5 Clinical validity: correlation of Dutch SWALQoL with Euroqol

a Significant at 0.01 level (2-tailed)

b Significant at 0.05 level (2-tailed)

a Significant at 0.01 level
(2-tailed)
b Significant at 0.05 level
(2-tailed)

\section{Discussion}

In our study we made a cross-cultural adaptation of SWALQoL and validated this assessment tool against Euroqol in 152 Dutch patients with dysphagia. None of the subscales of SWAL-QoL-NL reached an internal consistency of 0.95 or higher. Nine subscale scores (General burden, Food selection, Eating duration, Fear of eating, Sleep, Fatigue, Mental health, Social functioning and a symptom score) ranged between 0.80 and 0.92 (Cronbach's $\alpha$ ) and can be used for assessment of quality of life in dysphagic patients on a group level. Two subscales (Eating desire and Communication scored only 0.67 and 0.60 , respectively, and are less suitable for clinical research. In the final version of SWALQoL-NL these two subscales with three and two items, respectively, were removed, reducing the questionnaire to a 39-item tool.

In literature it is described that there are differences in health-related quality of life measures between different cultures. In 1988 Flaherty et al. [17] already described that researchers should give particular attention to cross-cultural validity when an instrument designed in one culture is used in a second culture. Consequently, in 1993 Guillemin et al. [1] described their guidelines for cross-cultural adaptation of health-related quality-of-life measures based on a systematic review of translated questionnaires. Our study shows that some cultural differences seem to exist between dysphagia patients in the U.S. and in the Netherlands. This supports the findings of Guillemin et al. [1] that one should thoroughly investigate a translated questionnaire clinimetrically before using it in another language. Although the subscales Eating desire and Communication are valid in the original version of SWAL-QoL, they are not psychometrically valid for use in the Netherlands. Researchers in the Netherlands should be aware of these findings when comparing their SWAL-QoL-NL outcomes with the outcomes measured by the original SWAL-QoL in other publications. Also, Dutch researchers should specifically address in their reports that they have used SWALQoL-NL in their research and not the original 44-item version.

Interestingly, the 14-item battery that provides clinical researchers and practitioners information on symptoms had an internal consistency of 0.80 , allowing the use of a sum score for these 14 items on a group level. We renamed this 14-item battery the Symptom score. This symptom score 
can be derived from the raw data in the same way as the other subscale scores are calculated. Although the symptom score can be used only on a group level, it allows researchers to represent a variety of clinical symptoms in one severity score ranging from 0 (worst situation) to 100 (no problems).

The weak correlation in general between SWAL-QoL-NL and Euroqol shows that both instruments are measuring two related but different constructs. The correlations between three SWAL-QoL-NL subscales (Eating duration, Social effects and Fatigue and the VAS score on Euroqol were found to be significantly correlated. The correlations for the first two constructs were found to be weak, but the correlation between the SWAL-QoL-NL subscale Fatigue and the VAS score on Euroqol $(r=0.36)$ can be marked as substantial $(r \geq 0.30)$ and satisfactory for group-level comparisons [15]. These findings support the hypothesis that there is a small overlap between the two quality-of-life questionnaires but that SWAL-QoL measures a very specific construct compared to more general quality-of-life issues. The three described correlations were significant at a 0.01 level. There were also three other significant correlations found, but only at a 0.05 level. These weak correlations might support the findings described above, but considering the fact that 66 correlations were calculated for Table 5, these significant findings might also be interpreted as a type 1 error.

Combined with the high internal consistency scores ( 0.80 and above), SWAL-QoL-NL can be considered a reliable tool to assess the effects of dysphagia on quality of life in different patient populations.

\section{Conclusion}

SWAL-QoL-NL proved to be a reliable tool to measure quality of life in patients with oropharyngeal dysphagia. Nine subscales can be used for assessment of quality of life in dysphagic patients on a group level. Two subscales (with a total of 5 items) could be removed from the item list, reducing the SWAL-QoL-NL to a 39-item tool. Interestingly, in the Dutch version a sum score can be derived from the items about clinical symptoms, allowing the use of a single score representing the severity of clinical symptoms.

Acknowledgments The authors thank Kathleen Gallagher and Tamar Gibson for their help in translating and back-translating the questionnaires.

Open Access This article is distributed under the terms of the Creative Commons Attribution Noncommercial License which permits any noncommercial use, distribution, and reproduction in any medium, provided the original author(s) and source are credited.

\section{References}

1. Guillemin F, Bombardier C, Beaton D. Cross-cultural adaptation of health-related quality of life measures: literature review and proposed guidelines. J Clin Epidemiol. 1993;46:1417-32.

2. Gustafsson B, Tibbling L. Dysphagia, an unrecognized handicap. Dysphagia. 1991;6:193-9.

3. McHorney CA, Bricker DE, Kramer AE, Rosenbek JC, Robbins J, Chignell KA, et al. The SWAL-QOL outcomes tool for oropharyngeal dysphagia in adults: I. Conceptual foundation and item development. Dysphagia. 2000;15:115-21.

4. McHorney CA, Bricker DE, Robbins J, Kramer AE, Rosenbek JC, Chignell KA. The SWAL-QOL outcomes tool for oropharyngeal dysphagia in adults: II. Item reduction and preliminary scaling. Dysphagia. 2000;15:122-33.

5. Bland JM, Altman DG. Cronbach's alpha. BMJ. 1997;314:572.

6. McHorney CA, Robbins J, Lomax K, Rosenbek JC, Chignell K, Kramer AE, et al. The SWAL-QOL and SWAL-CARE outcomes tool for oropharyngeal dysphagia in adults: III. Documentation of reliability and validity. Dysphagia. 2002;17:97-114.

7. Lovell SJ, Wong HB, Loh KS, Ngo RY, Wilson JA. Impact of dysphagia on quality-of-life in nasopharyngeal carcinoma. Head Neck. 2005;27:864-72.

8. Costa Bandeira AK, Azevedo EH, Vartanian JG, Nishimoto IN, Kowalski LP, Carrara-de Angelis E. Quality of life related to swallowing after tongue cancer treatment. Dysphagia. 2008;23:183-92.

9. Roe JW, Leslie P, Drinnan MJ. Oropharyngeal dysphagia: the experience of patients with non-head and neck cancers receiving specialist palliative care. Palliat Med. 2007;21:567-74.

10. Euroqol Group, EuroQol—a new facility for the measurement of health-related quality of life. The EuroQol Group. Health Policy. 1990;16:199-208.

11. Furtado K, Pina E, Moffatt CJ, Franks PJ. Leg ulceration in Portugal: quality of life. Int Wound J. 2008;5:34-9.

12. Knoll GA, Cantarovitch M, Cole E, Gill J, Gourishankar S, Holland D, et al. The Canadian ACE-inhibitor trial to improve renal outcomes and patient survival in kidney transplantation study design. Nephrol Dial Transplant. 2008;23:354-8.

13. Ulvik A, Kvale R, Wentzel-Larsen T, Flaatten H. Quality of life 2-7 years after major trauma. Acta Anaesthesiol Scand. 2008;52:195-201.

14. McDowell I, Newell C. Measuring health. A guide to rating scales and questionnaires. New York, Oxford: Oxford University Press, 1996.

15. Aday LA, Designing and conducting health surveys. San Francisco: Jossey-Bass Publishers, 1996.

16. DeVellis RF, Scale development. Theory and applications. Thousand Oaks, CA: Sage Publications; 2001.

17. Flaherty JA, Gaviria FM, Pathak D, Mitchell T, Wintrob R, Richman JA, et al. Developing instruments for crosscultural psychiatric research. J Nervous Mental Dis. 1988;176:257-63.

H. C. A. Bogaardt Msc

R. Speyer $\mathrm{PhD}$

L. W. J. Baijens MD

W. J. Fokkens MD, $\mathrm{PhD}$ 